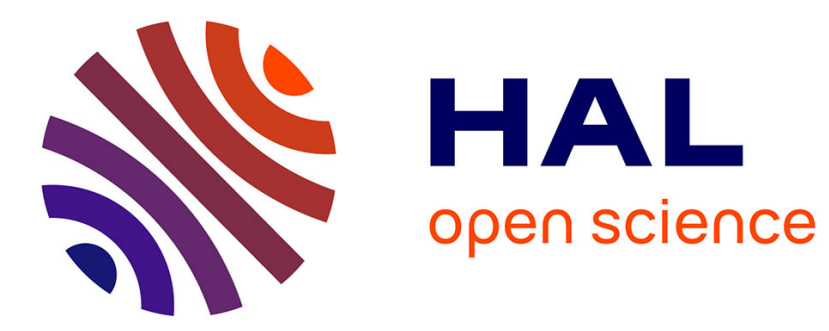

\title{
Un exemple de conservation du patrimoine génétique chez la poule
}

Eh. Ricard, M.J. Petitjean

\section{To cite this version:}

Eh. Ricard, M.J. Petitjean. Un exemple de conservation du patrimoine génétique chez la poule. Productions Animales, 1988, 1 (5), pp.345-354. hal-00895847

\section{HAL Id: hal-00895847 https://hal.science/hal-00895847}

Submitted on 1 Jan 1988

HAL is a multi-disciplinary open access archive for the deposit and dissemination of scientific research documents, whether they are published or not. The documents may come from teaching and research institutions in France or abroad, or from public or private research centers.
L'archive ouverte pluridisciplinaire HAL, est destinée au dépôt et à la diffusion de documents scientifiques de niveau recherche, publiés ou non, émanant des établissements d'enseignement et de recherche français ou étrangers, des laboratoires publics ou privés. 
INRA Prod. Anim., 1988, 1 (5), 345-354

\section{F.H. RICARD, M.J. PETITJEAN*}

INRA

Station de Recherches Avicoles Nouzilly 37380 Monnaie

${ }^{*}$ INRA Domaine de Magneraud Laboratoire de Spermiologie 17700 Surgères

\section{Un exemple de conservation du patrimoine génétique chez la poule}

L'élevage avicole intensif n'utilise qu'un très petit nombre de souches
qui sont soumises à de très fortes pressions de sélection. Mais il existe,
au sein même des firmes de sélection, et dans divers Centres
de Recherches, des populations qui présentent des caractéristiques
originales et qui peuvent convenir pour occuper des créneaux particuliers.

L'amélioration génétique des animaux domestiques a abouti, chez toutes les espèces, à la création de souches ou lignées bien caractérisées quant à leurs aptitudes zootechniques. Mais l'évolution moderne tend à ne conserver que les plus performantes d'entre elles qui sont alors très largement utilisées (exemples: bovins de race Frisonne, poulets de type Cornish) au détriment de toutes les autres. La crainte d'une disparition pure et simple de ces dernières, c'est-à-dire la perte d'une part importante de la variabilité géné-

\footnotetext{
Résumé

Une action de conservation génétique a été entreprise sur 4 souches de poules créées à la Station INRA du Magneraud en vue d'être utilisées dans des croisements commerciaux mais dont la sélection selon cette optique a été abandonnée en 1977. Leur nom de code et les principales caractéristiques sont les suivantes : M-22, une Wyandotte blanche à crête simple; $\mathrm{M-33}$, souche synthétique provenant pour moitié de la race Marans ; M-88, souche synthétique à base des races Wyandotte et Rhode-Island et porteuse du gène de nanisme $\mathrm{dw} ; \mathrm{P}-00$, une Leghorn blanche à crête en pois et pondant des oufs à coquille bleutée.

Le programme de conservation prévoyait une pression de sélection légère et un contrôle détaillé des performances. Pour la sélection, $\mathbf{5 0} \%$ des poules contrôlées étaient gardées comme reproductrices sur la base du nombre d'œufs commercialisables pondus à l'âge de 42 semaines. Les résultats observés au cours de 5 générations obtenues de 1981 à 1985 sont présentés et discutés dans le présent article.

La meilleure pondeuse s'est révélée être la souche P-00, suivie de la souche M-22. Les 2 souches M-22 et M-33, qui avaient des performances de ponte comparables aux souches nanifiées de reproductrices chair commerciales, pourraient être utilisées dans la production d'un poulet de type « label ». La souche M-33, qui donne des cufs à coquille brun foncé, pourrait intéresser tous ceux qui recherchent une coquille très colorée. La souche M-88 présentait le moins bon taux de ponte et le plus fort pourcentage d'œufs cassés, mais le poids de l'œuf était élevé malgré la présence du gène de nanisme. Un effort avait été entrepris pour améliorer la solidité de la coquille de ses ceufs, il faudrait le poursuivre.
}

tique, a poussé de nombreux scientifiques à insister pour qu'une action de conservation de ce patrimoine soit entreprise (Boyer, 1965 ; Merat, 1972 ; Cauderon, 1980).

En ce qui concerne les volailles, le maintien des anciennes races françaises, créées et/ou décrites principalement au cours du XIX siècle, préoccupe depuis toujours les milieux avicoles traditionnels. Boyer (1965) avait proposé que les pouvoirs publics aident financièrement les éleveurs non professionnels qui s'engageraient à conserver de petits troupeaux de volailles, à les faire se reproduire et à assurer un minimum de contrôles zootechniques. Cette idée s'est concrétisée en 1978 avec la création, sous l'égide du ministère de l'Agriculture, d'un Conservatoire national des races d'animaux de basse-cour (Arnold et de Rochambeau, 1983). Une autre idée consiste à maintenir en ségrégation dans une même population le maximum de gènes connus, et à étudier leurs effets sur différentes caractéristiques d'intérêt économique. Pour l'espèce poule, un troupeau de ce type a été mis en place par Merat (1962) au Centre INRA de Jouy-en-Josas. On peut ensuite utiliser ces animaux pour transférer des allèles jugés intéressants dans les souches commerciales. Une troisième attitude consiste à s'intéresser aux souches en tant qu'entité génotypique. Il peut s'agir de lignées expérimentales correspondant à un modèle biologique, par exemple deux lignées qui ont été sélectionnées de façon divergente pour une caractéristique physiologique donnée. Il peut s'agir aussi de souches commerciales qui ne sont plus utilisées à grande échelle soit parce que leur niveau de performance est devenu insuffisant, soit parce que leur génotype ne correspond pas, ou ne correspond plus, à ce qui est demandé. Or, ces souches gardent de l'intérêt parce qu'elles sont homogènes, qu'elles 
possèdent un génotype équilibré et stable, qu'elles ont quelquefois des caractéristiques originales, et qu'elles peuvent garder un niveau de production satisfaisant. Par ailleurs, ce type de souches peut intéresser une fraction de la profession avicole qui cherche à produire des animaux différents du modèle stanctard. Elles peuvent également correspondre à la demande d'organismes qui ne sont pas soumis aux nécessités de la performance maximum, par exemple dans les pays en voie de développemenl.

Nous nous sommes intéressés à ce troisième type de conservation à propos des souches de poules créées à la Station INRA du Magneraud par Cochez en vue d'entrer dans les croisements qui ont été commercialisés jusqu'en 1976. Par la suile, l'INRA a maintenu pendant plusieurs générations en conservatoire génétique les 4 souches de pondeuses les plus originales du Magneraud, en poursuivant une sélection « légère " pour, au minimum, maintenir le niveau de production et contrôler ce niveau au cours des générations de conservation. Dans le présent article, nous présentons les caractéristiques du travail accompli et analysons les résultats observés sur les animaux nés de 1981 à 1985 et contrôlés jusqu'au mois d'octobre 1986.

\section{1 / Conditions expérimentales}

\section{1 / Origine des souches conservées}

Les 4 souches sont désignées par leur numéro de code technique tel qu'il était utilisé dans l'organisation de la sélection au Magneraud : $\mathrm{M}-22$, M-33, M-88 et P-00. Jusqu'en 1978, chacune correspondait à une population de reproducteurs de 20 coqs et de 200 poules.

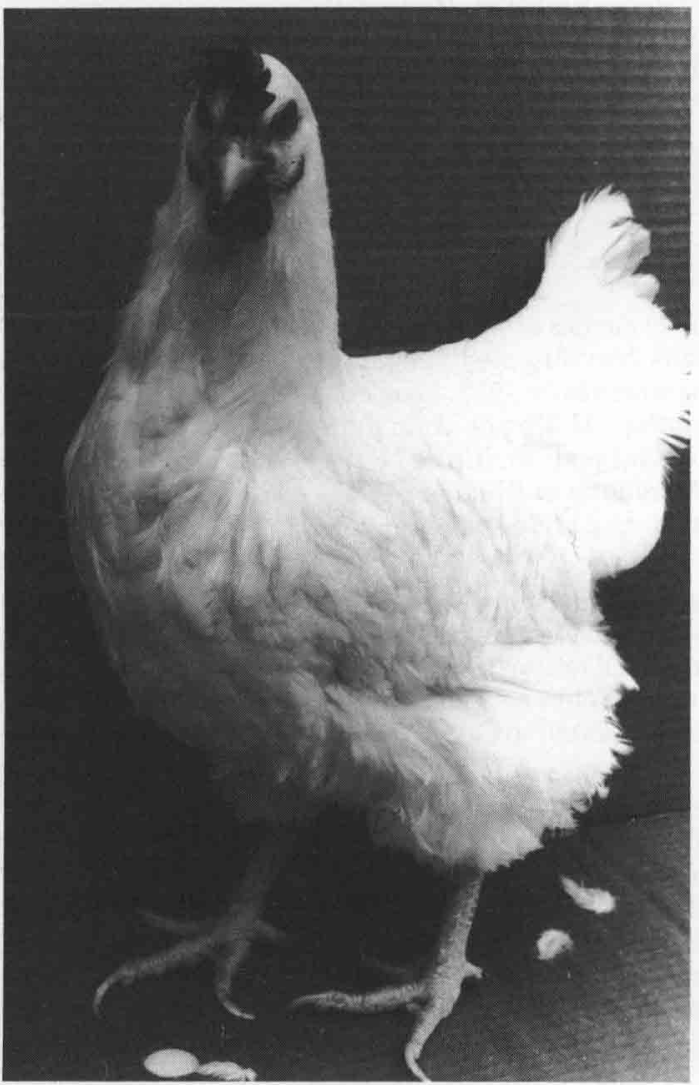

\section{a / La souche $M-22$}

Elle a pour origine la race $W_{\text {vandotte blanche à }}$ crête rosacée de l'élevage SARGAS (Cochez, 1951). L'allèle $r$, correspondant à la crête simple, a ensuite été introduit afin d'éliminer le facteur de subfertilité du sperme lié à l'allèle $R$ (Petitjean et Cochez, 1966). Au point de vue génotypique, la souche M-22 est homozygote pour la crête simple (formule rrpp), les pattes jaunes (formule $w w I-$ $d I d$ ) et le plumage blanc (formule $c c$, c'est-à-dire blanc récessif). Elle était sélectionnée par Cochez comme pondeuse d'œufs à coquille colorée (brun clair).

\section{b / La souche $M-33$}

Elle a pour origine un croisement réalisé en 1965 entre une souche de race Marans à plumage roux (allèle $s$, récessif lié au sexe) et une souche créée à la Station de Recherches Avicoles de Jouyen-Josas à partir de croisements faisant intervenir la race Gatinaise (Merat, 1955). Au point de vue génotypique, elle est homozygote par la crête simple, les pattes jaunes et un plumage roux et noir. C'est une pondeuse d'œufs à coquille brun foncé, comme la race Marans mais avec une variabilité de couleur plus grande.

\section{C / La souche M-88}

Il s'agit d'une souche synthétique créée par Cochez à partir des races Marans coucou argentée, Rhode-Island rouge et Wyandotte blanche, d'abord sélectionnée comme pondeuse de gros œufs, et dans laquelle a été introduit le gène de nanisme lié au sexe $d w$. Les lignées de $W$ yandotte et de Rhode-Island utilisées avaient été sélectionnées pour augmenter leur vitesse de croissance. La souche M-88 possède une crête simple, des pattes jaunes et un plumage qui ressemble à celui de la race New-Hampshire. Elle pond des oufs à coquille colorée.

\section{d / La souche P-00}

Elle a pour origine une population de Leghorn blanche (Elevage des Peupliers, 37380 Monnaie) dans laquelle ont été introduits les allèles $P$ (crête en pois) et $O$ (œuf bleu) originaires de la population polymorphe de Jouy-en-Josas (Silbert et Merat, 1974) eux-mêmes provenant de la race Araucana. En dehors de ces 2 gènes, le phénotype Leghorn a été maintenu, en particulier la patte jaune et le plumage blanc (génotype $I I$, c'est-àdire blanc dominant). La souche P-00 est une pondeuse d'œufs à coquille bleutée.

\section{2 / Obtention de cheptels étudiés et conditions d'élevage des animaux}

Au mois de mai 1981, les poussins des souches M-22, M-33 et P-00 ont été obtenus à partir de reproducteurs éclos au mois d'août 1979. A la même date, les poussins de la souche M-88 ont été obtenus à partir de reproducteurs éclos en 1978 et qui avaient subi une mue forcée au cours de l'été 1980. Ces animaux, éclos en mai 1981, ont fourni les reproducteurs qui ont donné les poussins de la génération née en octobre 1982. Par la suite, une reproduction a été faite chaque année, les écolosions ayant lieu au mois d'octobre. 
Les poussins étaient élevés sur litière de copeaux de bois dans un bâtiment sans fenêtre, avec $14 \mathrm{~h}$ de lumière ef $10 \mathrm{~h}$ d'obscurité jusqu'à l'âge de 18 semaines. Ils étaient ensuite placés en cages individuelles dans une même cellule d'un poulailler très bien protégé des aléas climatiques, avec 14 heures de lumière par jour. L'aliment, donné sous forme de farine, comprenait 3 formules dont les caractéristiques principales étaient les suivantes : $2850 \mathrm{kcal}$ d'énergie métabolisable et $190 \mathrm{~g}$ de matières azotées totales (MAT) par kg entre 0 et 9 semaines d'âge, 2800 kcal et $160 \mathrm{~g}$ de MAT entre 9 et 20 semaines, 2750 $\mathrm{kcal}$ et $165 \mathrm{~g}$ de MAT au-delà de 20 semaines d'âge.

L'objectif était de faire éclore à chaque génération 400 poussins (sexes mélangés) par souche et de conserver 40 à 50 cocjs et 150 poules parmi lesquels devaient être sélectionnés les reproducteurs de la génération suivante. Les taux d'éclosion (obtenus en parquets pédigrés) sont indiqués dans la figure 1. Les chiffres relativement faibles observés en 1981 et 1982 sont dus à l'âge élevé des reproducteurs (la souche M-22 a toutefois présenté un taux d'éclosion correct). C'est l'une des raisons qui nous ont amené par la suite à prévoir une reproduction tous les ans, les dates étant choisies en fonction des impératifs de travail de la Station du Magneraud. Les effectifs totaux de poussins éclos (sexes mélangés) et de poules placées en batterie pour les contrôles de ponte sont visualisés dans la figure 2 .

\section{3 / Conditions de contrôle et de sélection des reproducteurs}

\section{a / Choix des poules}

Les effectifs de poules effectivement placées dans la batterie de ponte représentaient entre 50 et $90 \%$ des poussins femelles éclos et mis en élevage : moyenne globale de $72 \%$ pour les souches M-22 et M-33, $78 \%$ pour la souche $M-88$ et $68 \%$ pour la souche P-00. Le choix était fait, compte tenu d'une mortalité juvénile relativement faible, de façon à avoir en contrôle de ponte 2 à 3 poules par famille (une famille : descendance d'une poule accouplée à un seul coq selon le schéma " pédigré » classique de la sélection avicole).

Les animaux étaient en cages individuelles, ce qui a permis de contrôler aisément la ponte de chaque poule. On a noté séparément les œufs " cassés » (incluant égalementles œufs à coquille fêlée, les oufs doubles, mous, hardés, ou anormalement petits). La batterie de ponte utilisée avait la particularité d'être très rigide et d'entraîner un fort pourcentage d'œufs cassés. Nous avions ainsi une bonne estimation de la solidité de la coquille et nous avons pu tenir compte de ce caractère dans le programme de sélection. Les totaux d'œufs (normaux, cassé et totaux) étaient calculés et cumulés par périodes de 2 semaines. Les contrôles ont été effectués jusqu'à l'âge de 61 semaines pour les poules nées en 1981, 42 semaines pour les poules nées en 1982, 50 semaines pour les poules nées en 1984 et 53 semaines pour les poules nées en 1983 et 1985.

Le critère de sélection des reproductrices était le nombre total d'œufs normaux pondus à l'âge
Figure 1. Taux d'éclosion (poussins nés/ceufs mis en incubation, en \%) par souche et par année entre 1981 et 1985. Les moyennes par souche sont indiquées entre parenthèses.

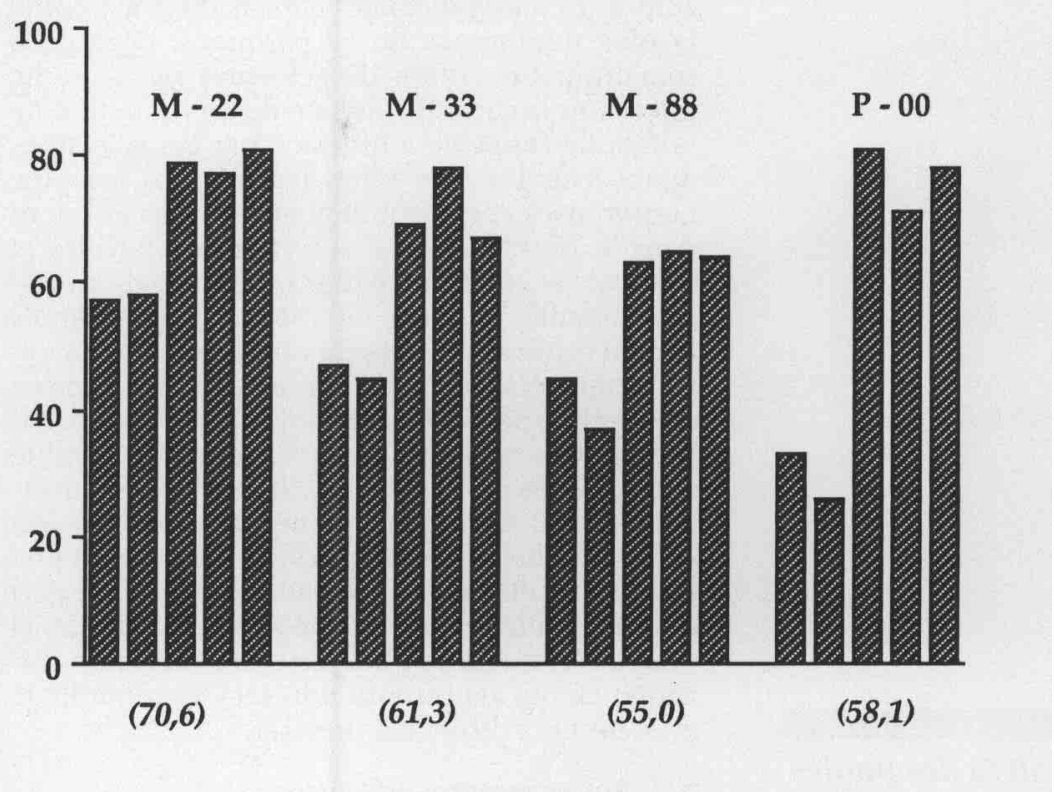

Figure 2. Effectifs de poussins éclos et de poules placées en batteries de ponte, par souche et par année, de 1981 à 1985.

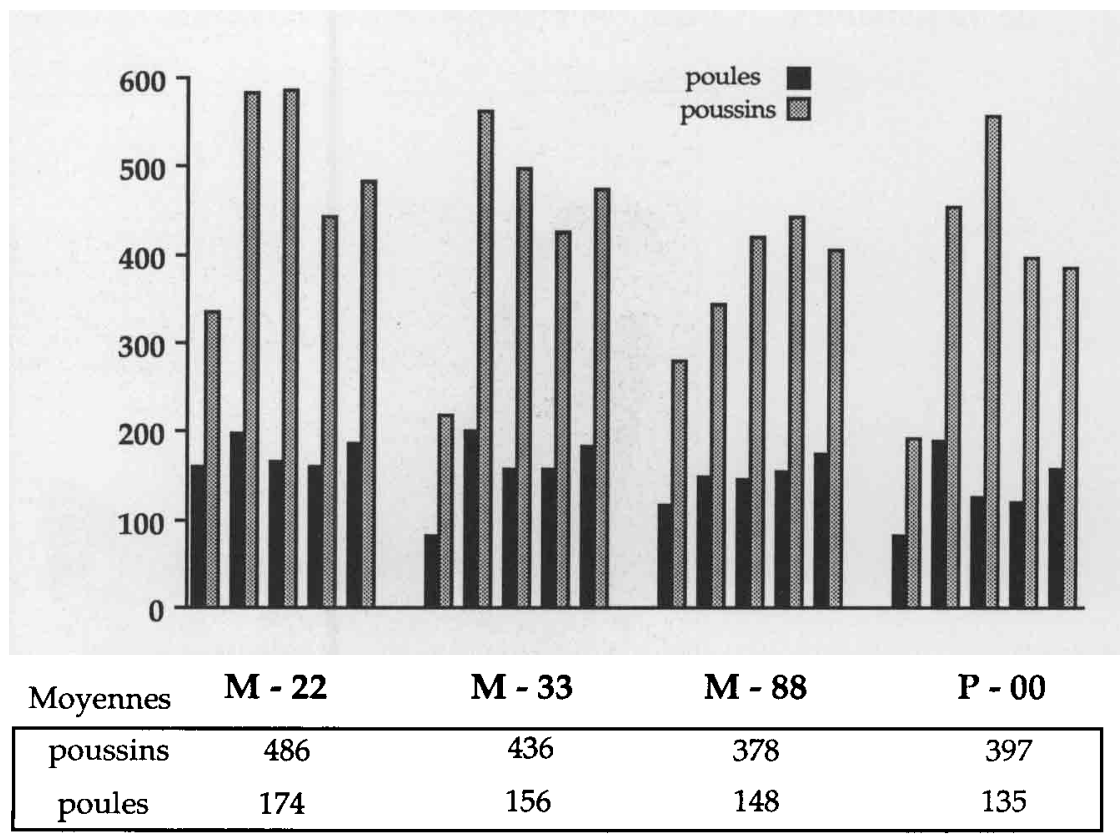

de 42 semaines. Ce critère faisait donc intervenir à la fois l'aptitude de ponte et la solidité de la coquille. Nous avons de cette façon retenu 80 poules pour chaque souche qui ont été réparties en 16 groupes pédigrés de $1 \mathrm{coq} \times 4$ ou 5 poules, chaque reproducteur d'un même groupe étant issu d'un père différent. En dehors de cette contrainte, les appariements étaient faits au hasard. La reproduction était réalisée par insémination artificielle. La technique utilisée se rapprochait donc d'une sélection massale avec un taux de sélection de $50 \%$. 


\section{b / Choix des coqs}

L'objectif était de garder 3 coqs par parquet pédigré, soit théoriquement 48 par souche. Les coqs d'un parquet étaient choisis dans la famille la plus nombreuse de ce parquet à l'âge de 8 semaines. Ce critère de sélection faisait donc intervenir la capacité globale de reproduction de la famille (appelée "fitness » par les sélectionneurs avicoles). En effet, les familles les plus nombreuses correspondent aux poules qui pondent le plus, aux meilleurs taux de fertilité et d'éclosivité, ainsi qu'au plus faible taux de mortalité juvénile. A partir de 1983, les coqs gardés étaient testés sur la base du nombre de spermatozoïdes par éjaculat et de leur motilité : le nombre était estimé par le produit du volume et de la densité optique mesurée au photomètre ; la motilité était estimée après environ 18 heures de conservation à $+3^{\circ} \mathrm{C}$ à l'aide d'un microscope associé à un appareillage photoélectrique fournissant une note pouvant varier de 0 à 80 . Dans chaque parquet le meilleur donneur de sperme était choisi pour assurer la reproduction à la génération suivante, ce qui représente une sélection familiale avec un taux difficile à préciser.

$50 \%$ des poules contrôlées sont gardées comme reproductrices sur la base du nombre total d'wufs normaux pondus à l'âge de 42 semaines.

\section{c / Autres mesures effectuées}

Le poids vif des animaux a été déterminé aux stades juvénile et/ou adulte à chaque génération de façon à pouvoir comparer les performances de croissance des 4 souches.

La précocité sexuelle des poules a été estimée individuellement par l'âge (en jours) au premier œeuf contrôlé, que celui-ci soit normal ou cassé.

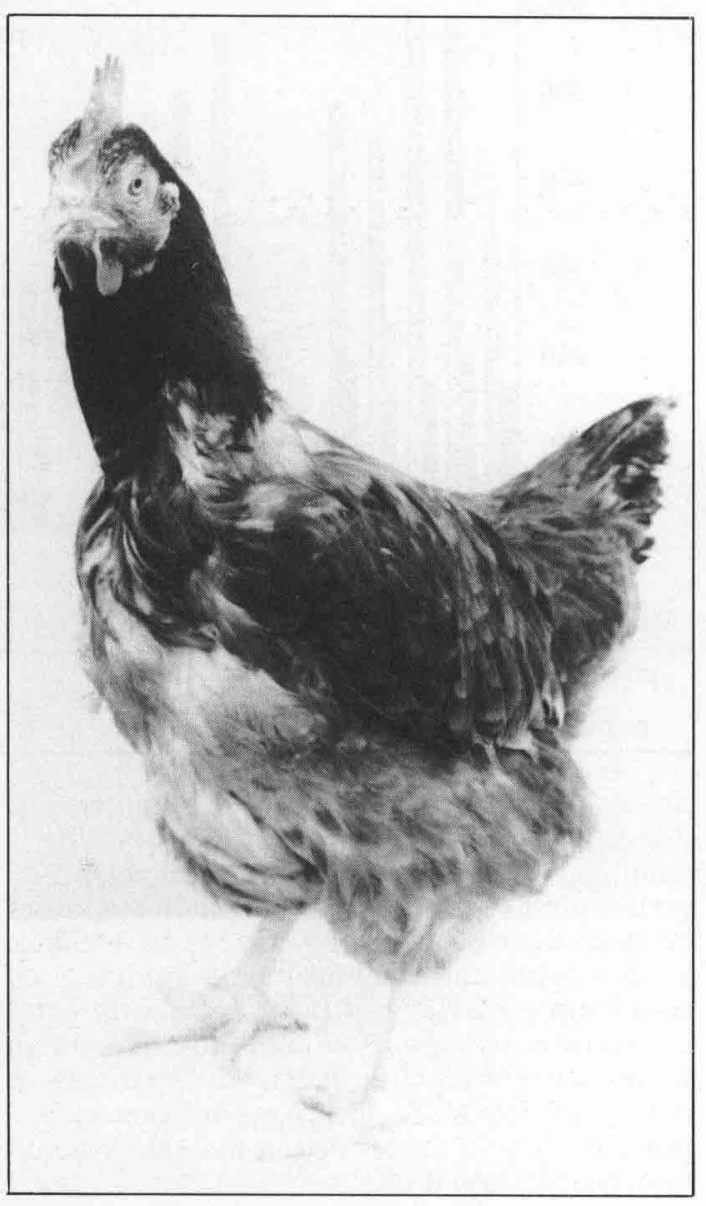

Un contrôle du poids de l'œuf a été effectué par pesée de la ponte individuelle de chaque poule durant 2 semaines consécutives (pour les générations 1981, 1983 et 1984) ou par pesée globale par souche d'une journée de ponte (pour la génération 1985). L'âge des poules étant variable selon les générations, les résultats doivent être considérés comme issus d'échantillons particuliers ne permettant qu'une comparaison entre souches.

\section{4 / Calculs réalisés}

De nombreux caractères ont été mesurés individuellement ce qui a permis de calculer les écarts-types et d'effectuer des analyses de variance: soit pour tester l'effet souche (intraannée d'éclosion), soit pour tester l'effet génération de sélection (c'est-à-dire l'effet année d'éclosion intra-souche), soit pour tester l'effet combiné souche $\times$ génération de sélection. Une comparaison multiple de moyennes a ensuite été faite en utilisant le test de Newmann et Keuls. Les effectifs variaient selon les souches et les années (de 80 à 180 pour les poules contrôlées en cages individuelles). En conséquence, les analyses ont été faites en considérant que l'effectif était le même pour toutes les variantes du traitement étudié, effectif égal au plus petit d'entre eux. Par contre, les moyennes et écarts-types utilisés étaient ceux calculés à partir de tous les individus contrôlés et ont servi à recalculer les carrés moyens basés sur des degrés de liberté correspondant aux effectifs communs retenus. Cette technique entraîne une diminution du nombre total de degrés de liberté pour la variance résiduelle, mais elle permet d'interpréter les interactions avec beaucoup plus de sûreté, ce qui est primordial dans une expérimentation zootechnique.

Pour les taux de mortalité et d'œufs cassés nous avons calculé une valeur globale pour chaque souche et les comparaisons ont été faites en utilisant le test $x^{2}$.

Enfin, les comparaisons entre souches ont été faites à âge égal, exprimé en semaines \pm 2 jours. Comme les contrôles de ponte étaient totalisés par périodes de 2 semaines, et que toutes les souches n'éclosaient pas le même jour (généralement 2 groupes, $\mathrm{M}-22+\mathrm{M}-33$ et $\mathrm{M}-88+\mathrm{P}-00$, séparés par une semaine), les résultats ont été obtenus par intrapolation entre deux périodes successives quand cela s'avérait nécessaire.

\section{2 / Résultats}

Les valeurs moyennes du nombre d'œufs normaux contrôlés à l'âge de 42 semaines, qui est le critère principal de sélection, sont détaillées dans le tableau 1. Ce nombre est systématiquement plus élevé pour la souche P-00 (en moyenne 107 œufs) et plus faible pour la souche M-88 (en moyenne 78 œufs]. L'écart-type du nombre d'œufs varie de 14 à 29, selon la souche et l'année d'éclosion, avec une valeur intra-groupe supérieure à 23 , ce qui représente un coefficient de variation de $25 \%$. La variation des valeurs relatives des 4 souches selon les années a entraîné une interaction souche $x$ année d'éclosion hautement significative $(\mathrm{P}<0,01)$. 
Tableau 1. Nombre d'œufs normaux contrôlés à l'âge de 42 semaines (= critère de sélection) par souche et par génération de sélection.

\begin{tabular}{|c|c|c|c|c|c|}
\hline \multirow{2}{*}{$\begin{array}{l}\text { Année } \\
\text { d'éclosion }\end{array}$} & \multicolumn{4}{|c|}{ Moyennes par souche } & \multirow{2}{*}{$\begin{array}{c}\text { Ecart-type } \\
\text { intra-souche } \\
\text { (1) }\end{array}$} \\
\hline & M-22 & M- 33 & M- 88 & P-00 & \\
\hline 1981 & $\begin{array}{c}88,5 \mathrm{~b}(2) \\
\mathrm{C}\end{array}$ & $\begin{array}{r}89,6 \mathrm{~b} \\
\mathrm{~B}\end{array}$ & $\begin{array}{r}77,7 \mathrm{c} \\
\mathrm{B}\end{array}$ & $\begin{array}{r}103,0 \mathrm{a} \\
\mathrm{B}\end{array}$ & 24,1 \\
\hline 1982 & $\begin{array}{r}96,0 \mathrm{~b} \\
\mathrm{~B}\end{array}$ & $\begin{array}{r}91,1 \mathrm{~b} \\
\mathrm{AB}\end{array}$ & $\begin{array}{r}67,3 \mathrm{c} \\
\mathrm{C}\end{array}$ & $\begin{array}{r}104,1 \text { a } \\
\text { B }\end{array}$ & 25,7 \\
\hline 1983 & $\begin{array}{r}97,5 \mathrm{a} \\
\mathrm{B}\end{array}$ & $\begin{array}{r}90,0 \mathrm{~b} \\
\mathrm{~B}\end{array}$ & $\begin{array}{r}79,6 \mathrm{c} \\
\mathrm{B}\end{array}$ & $\begin{array}{r}98,8 \text { a } \\
\text { B }\end{array}$ & 22,9 \\
\hline 1984 & $\begin{array}{r}104,1 \mathrm{~b} \\
\mathrm{~A}\end{array}$ & $\begin{array}{r}99,2 \mathrm{~b} \\
\mathrm{~A}\end{array}$ & $\begin{array}{r}76,7 \mathrm{c} \\
\mathrm{B}\end{array}$ & $\begin{array}{r}112,3 \text { a } \\
\mathrm{A}\end{array}$ & 22,7 \\
\hline 1985 & $\begin{array}{r}101,8 \mathrm{~b} \\
\mathrm{AB}\end{array}$ & $\begin{array}{r}96,6 \mathrm{C} \\
\mathrm{AB}\end{array}$ & $\begin{array}{r}87,1 \mathrm{~d} \\
\mathrm{~A}\end{array}$ & $\begin{array}{r}115,7 \text { a } \\
\mathrm{A}\end{array}$ & 22,2 \\
\hline
\end{tabular}

(1) Ecart-type intra-souche = racine carrée du carré moyen résiduel obtenu dans les analyses de variance entre souches intraannées.

(2) Résultat des tests de Newman et Keuls réalisés après analyse de variance à un facteur. Pour une ligne donnée, deux moyennes suivies d'une même lettre minuscule ne sont pas significativement différentes $(\mathrm{P}>0,05)$. Pour une colonne donnée, deux moyennes suivies d'une même lettre majuscule ne sont pas significativement différentes ( $\mathrm{P}>0,05)$. La lettre a (ou A) correspond au résultat le plus favorable, ici le plus grand nombre d'œufs.

Les résultats des pesées d'animaux vivants sont rassemblés dans le tableau 2, séparément pour les mâles et pour les femelles. Les poids juvéniles des années 1983 à 1985 ont été regroupés car tous les animaux ont été pesés au même âge. On observe systématiquement l'ordre décroissant suivant: $\mathrm{M}-33, \mathrm{M}-22, \mathrm{M}-88, \mathrm{P}-00$. Bien que porteuse du gène de nanisme $d w$, la souche M-88 donne des poulets relativement gros puisqu'ils atteignent 80 à $90 \%$ du poids de la souche la plus lourde (M-33). L'explication est à rechercher dans les caractéristiques génétiques de la population qui a été nanifiée.

Les effectifs de poules présentes aux différents âges permettent de calculer un taux de mortalité au cours de la saison de ponte. Afin d'avoir des effectifs suffisamment importants, nous avons regroupé l'ensemble des générations et les résultats sont indiqués dans le tableau 3, séparément pour les périodes 21-42 et 21-53 semaines d'âge. Dans les 2 cas, c'est la souche P-00 qui présente le plus faible taux de mortalité et la souche M-88 le plus fort. Il apparaît qu'entre 42 et 53 semaines d'âge (soit 11 semaines) la mortalité est aussi forte qu'entre 21 et 42 semaines (soit 21 semaines). Les autopsies pratiquées sur tous les animaux morts, ont montré que le fort taux de mortalité des poules adultes M-88 était dû en grande partie à des problèmes de foie gras hémorragiques.

La précocité sexuelle a été estimée par l'âge au premier œuf contrôlé et les résultats sont détaillés dans le tableau 4. En moyenne, la souche P-00 est la plus précoce (premier œuf à 158 jours) et la souche M-88 la plus tardive (165 jours), l'écart étant d'une semaine. L'écart-type des groupes élémentaires souche-année varie de 4 à 19 jours, la valeur moyenne étant de 12 jours soit un coefficient de variation un peu supérieur à $7 \%$. L'évolution en fonction du numéro de génération est irrégulière, ce qui suggère l'existence d'un effet important des conditions d'élevage sur ce caractère, en particulier pour les poules nées en 1983. Mais cette irrégularité n'est pas la même selon les souches et ceci entraîne une interaction souche $\times$ année d'éclosion hautement significative.

Tableau 2. Poids vif des animaux, en grammes, mesuré à différents âges.

\begin{tabular}{|c|c|c|c|c|c|c|}
\hline \multirow{2}{*}{$\begin{array}{c}\text { Année } \\
\text { d'éclosion }\end{array}$} & \multirow{2}{*}{$\begin{array}{c}\text { Age } \\
\text { [semaines) }\end{array}$} & \multicolumn{4}{|c|}{ Moyennes par souche } & \multirow{2}{*}{$\begin{array}{l}\text { Ecart-type } \\
\text { intra-souche }\end{array}$} \\
\hline & & M-22 & M- 33 & M- 88 & P-00 & \\
\hline 1) Mâles & & & & & & \\
\hline $1982(2)$ & 13 & $1972 b$ & $2084 \mathrm{a}$ & $1653 \mathrm{c}$ & $1536 \mathrm{~d}$ & 179 \\
\hline 1983-1985 & 8 & $1061 \mathrm{~b}$ & $1174 \mathrm{a}$ & $1003 \mathrm{c}$ & $866 \mathrm{~d}$ & 91 \\
\hline 2) Femelles & & & & & & \\
\hline $1977(1)$ & 36 & 2197 & 2272 & 1838 & 1810 & - \\
\hline 1981 & 18 & $1656 \mathrm{~b}$ & $1773 a$ & $1424 \mathrm{C}$ & $1330 \mathrm{~d}$ & 130 \\
\hline $1982(2)$ & 13 & $1452 \mathrm{~b}$ & $1512 \mathrm{a}$ & $1216 \mathrm{c}$ & $1146 \mathrm{~d}$ & 103 \\
\hline 1983-1985 & 8 & $825 \mathrm{~b}$ & 899 a & $781 \mathrm{c}$ & $699 \mathrm{~d}$ & 73 \\
\hline $1985(3)$ & 50 & $2458 \mathrm{~b}$ & $2691 \mathrm{a}$ & $2404 b$ & $1818 \mathrm{c}$ & 267 \\
\hline
\end{tabular}

(1) Pesées réalisées au cours du travail de sélection de L.P. Cochez (communication personnelle) avant la mise en place du conservatoire. Ecarts-types non disponibles.

(2) Poulets des 2 sexes et des 4 souches élevés dans une mêne case de poussinière.

(3) Estimation sur un échantillon de 15 poules par souche.

Les poules nées en 1985 ont ainsi pondu en moyenne 10 aufs de plus que celles nées en 1981 . 


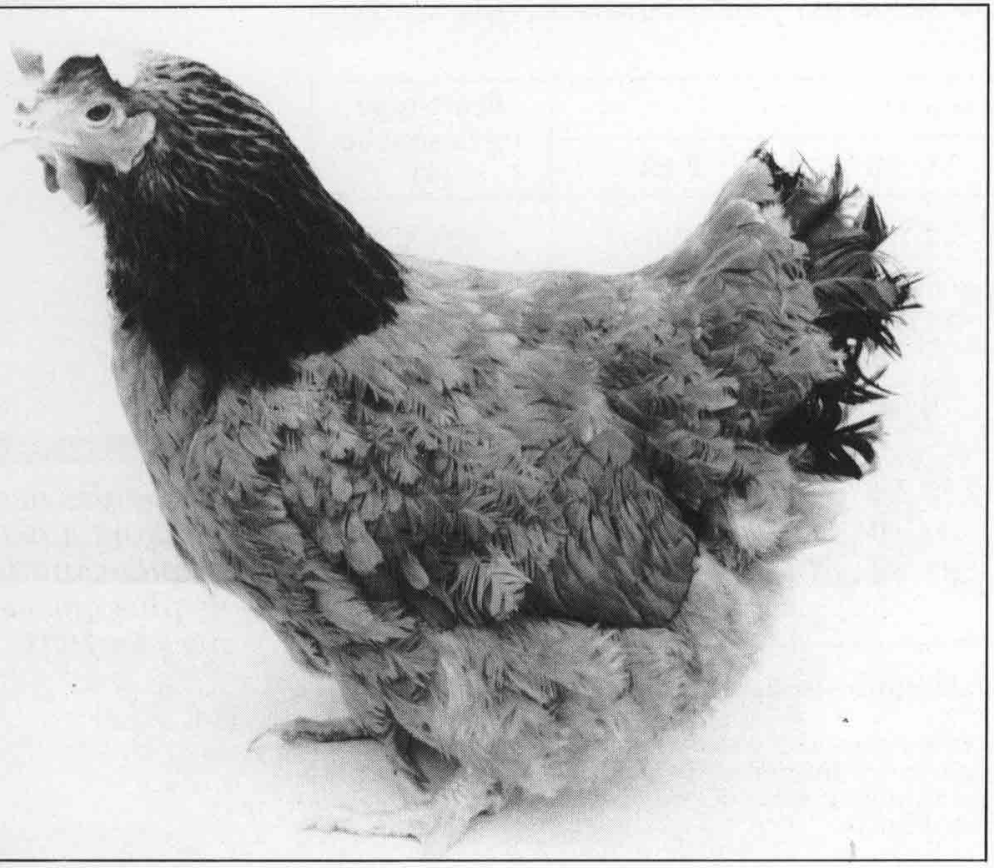

Poule de la souche M-88.
Les résultats concernant l'ensemble des œufs contrôlés (normaux + cassés + anormaux) sont détaillés dans le tableau 5 . Nous avons indiqué les valeurs observées à 42 semaines d'âge, qui existent pour chacune des 5 générations étudiées, ainsi que les valeurs observées en fin de contrôle, dont l'âge varie selon les générations. On observe globalement l'ordre décroissant suivant : P-00, M-22, M-33, M-88, la différence étant de 20 œufs entre les 2 extrêmes quand les poules sont âgées de 42 semaines. La ponte des poules M-22 est globalement supérieure $(\mathrm{P}<0,05)$ à celle des poules M-33, mais la valeur relative est inversée pour l'année 1981. Il en résulte une interaction significative $(\mathrm{P}<0,05)$ quand on fait une analyse de variance à 2 facteurs, la souche et l'année d'éclosion. Les valeurs relativement faibles observées en 1983 peuvent être reliées à une entrée en ponte plus tardive (cf tableau 4). Par ailleurs l'écart-type du nombre total d'œufs contrôlés à l'âge de 42 semaines (21) est plus faible que celui du nombre d'œufs normaux $(23,5)$. On obtient donc un coefficient de variation plus faible pour le nombre total d'œufs contrôlés (20\%) que pour le nombre d'œufs normaux $(25 \%)$. Cette différence provient du fait que certaines poules ont un petit nombre d'œufs normaux parce qu'elles ont beaucoup d'œufs cassés.

Les pourcentages d'œufs cassés (et anormaux) sont détaillés dans le tableau 6. Dans nos conditions d'élevage (batterie de ponte très rigide), les valeurs obtenues sont élevées, principalement pour la souche $\mathrm{M}-88$ qui présente donc les œufs à coquille la plus fragile. A partir de 1983 un effort a été fait pour améliorer la solidité de la coquille en éliminant les familles de poules M-88 qui présentaient les plus forts pourcentages d'œufs cassés, en plus du choix sur le nombre d'œufs normaux pondus par chaque poule. Les 2 souches M-22 et M-33 ont donné un fort pourcentage d'œufs cassés à la génération née en 1981, mais ce pourcentage a fortement diminué dès 1982, ce qui suggère un effet particulier de l'environnement en 1981 sans qu'il nous soit possible de préciser davantage. Enfin, la souche P-00 donne presque toujours le plus faible taux d'œufs cassés.

Tableau 3. Taux de mortalité pendant la ponte, par souche pour l'ensemble des années d'éclosion.

\begin{tabular}{|c|c|c|c|c|}
\hline \multirow{2}{*}{ Période } & \multicolumn{4}{|c|}{ Souche } \\
\cline { 2 - 5 } & \multicolumn{1}{|c|}{ M-22 } & M- 33 & M- 88 & P-00 \\
\hline $21^{\mathrm{e}}-42^{\mathrm{e}}$ semaine (1) & $2,2 \mathrm{a}(3)$ & $2,8 \mathrm{a}$ & $8,5 \mathrm{~b}$ & $1,6 \mathrm{a}$ \\
$21^{\mathrm{e}}-53^{\mathrm{e}}$ semaine (2) & $5,7 \mathrm{~b}$ & $5,7 \mathrm{~b}$ & $18,2 \mathrm{C}$ & $3,3 \mathrm{a}$ \\
\hline
\end{tabular}

(1) Ensemble des 5 années, 1981 à 1985

(2) Ensemble des 3 années, $1981+1983+1985$

(3) Comparaison des souches faites à l'aide du test $x^{2}$

Tableau 4. Précocité sexuelle des poules mesuree par lage (en jours) au premier cuf contrôlé. Les effectifs par groupe élémentaire souche $\times$ année varient de 83 à 196 .

\begin{tabular}{|c|c|c|c|c|c|}
\hline \multirow{2}{*}{$\begin{array}{c}\text { Année } \\
\text { d'éclosion }\end{array}$} & \multicolumn{4}{|c|}{ Moyennes par souche } & \multirow{2}{*}{$\begin{array}{l}\text { Ecart-type } \\
\text { intra-souche }\end{array}$} \\
\hline & M-22 & M- 33 & M- 88 & P-00 & \\
\hline 1981 & $\begin{array}{c}165 \mathrm{ab}(2) \\
\mathrm{C}\end{array}$ & $\begin{array}{r}163 \mathrm{a} \\
\mathrm{C}\end{array}$ & $\begin{array}{r}168 \mathrm{~b} \\
\mathrm{BC}\end{array}$ & $\begin{array}{r}163 \mathrm{a} \\
\mathrm{C}\end{array}$ & 14,3 \\
\hline 1982 & $\begin{array}{c}160 \mathrm{bc}(2) \\
\mathrm{B}\end{array}$ & $\begin{array}{r}158 \mathrm{~b} \\
\mathrm{~B}\end{array}$ & $\begin{array}{r}162 \mathrm{C} \\
\mathrm{A}\end{array}$ & $\begin{array}{r}152 \mathrm{a} \\
\mathrm{A}\end{array}$ & 13,2 \\
\hline 1983 & $\begin{array}{r}168 \mathrm{~b} \\
\mathrm{D}\end{array}$ & $\begin{array}{r}167 \mathrm{~b} \\
\mathrm{D}\end{array}$ & $\begin{array}{r}163 \mathrm{a} \\
\mathrm{AB}\end{array}$ & $164 \mathrm{a}$ & 6,6 \\
\hline 1984 & $\begin{array}{r}154 \mathrm{a} \\
\mathrm{A}\end{array}$ & $\begin{array}{r}152 \mathrm{a} \\
\mathrm{A}\end{array}$ & $\begin{array}{r}170 \mathrm{C} \\
\mathrm{C}\end{array}$ & $\begin{array}{r}159 \mathrm{~b} \\
\mathrm{~B}\end{array}$ & 12,9 \\
\hline 1985 & $\begin{array}{r}156 \mathrm{~b} \\
\mathrm{~A}\end{array}$ & $\begin{array}{r}153 \mathrm{a} \\
\mathrm{A}\end{array}$ & $\begin{array}{r}163 \mathrm{C} \\
\mathrm{AB}\end{array}$ & $\begin{array}{r}152 \text { a } \\
\text { A }\end{array}$ & 10,3 \\
\hline
\end{tabular}


Tableau 5. Nombre total d'œufs contrôlés selon l'âge des poules. Les effectifs par groupe élémentaire souche $\times$ année d'éclosion varient de 80 à 191 .

\begin{tabular}{|c|c|c|c|c|c|c|}
\hline \multirow{2}{*}{$\begin{array}{c}\text { Année } \\
\text { d'éclosion }\end{array}$} & \multirow{2}{*}{$\begin{array}{c}\text { Age } \\
\text { (semaines) }\end{array}$} & \multicolumn{4}{|c|}{ Moyennes par souche } & \multirow{2}{*}{$\begin{array}{l}\text { Ecart-type } \\
\text { intra-souche }\end{array}$} \\
\hline & & M-22 & $\mathbf{M}-\mathbf{3 3}$ & M- 88 & P-00 & \\
\hline 1981 & $\begin{array}{l}42 \\
\\
53 \\
61\end{array}$ & $\begin{array}{r}103 \mathrm{a} \\
\mathrm{B} \\
146 \mathrm{~b} \\
181 \mathrm{~b}\end{array}$ & $\begin{array}{r}105 \mathrm{a} \\
\mathrm{A} \\
151 \mathrm{~b} \\
187 \mathrm{~b}\end{array}$ & $\begin{array}{r}94 \mathrm{~b} \\
\mathrm{~B} \\
132 \mathrm{C} \\
158 \mathrm{C}\end{array}$ & $\begin{array}{r}108 \mathrm{a} \\
\mathrm{B} \\
163 \mathrm{a} \\
201 \mathrm{a}\end{array}$ & $\begin{array}{l}19,5 \\
\\
27,5 \\
35,3\end{array}$ \\
\hline 1982 & 42 & $\begin{array}{r}101 \mathrm{~b} \\
\mathrm{~B}\end{array}$ & $\begin{array}{r}100 \mathrm{~b} \\
\mathrm{AB}\end{array}$ & $\begin{array}{r}85 \mathrm{C} \\
\mathrm{B}\end{array}$ & $\begin{array}{r}109 \mathrm{a} \\
\mathrm{B}\end{array}$ & 24,1 \\
\hline 1983 & $\begin{array}{l}42 \\
53\end{array}$ & $\begin{array}{c}100 \mathrm{ab} \\
\mathrm{B} \\
149 \mathrm{a}\end{array}$ & $\begin{array}{r}95 \mathrm{~b} \\
\mathrm{~B} \\
141 \mathrm{~b}\end{array}$ & $\begin{array}{r}94 \mathrm{C} \\
\mathrm{A} \\
130 \mathrm{C}\end{array}$ & $\begin{array}{r}105 \mathrm{a} \\
\mathrm{B} \\
152 \mathrm{a}\end{array}$ & $\begin{array}{l}21,0 \\
31,3\end{array}$ \\
\hline 1984 & $\begin{array}{l}42 \\
50\end{array}$ & $\begin{array}{r}109 \mathrm{~b} \\
\mathrm{~A} \\
140 \mathrm{~b}\end{array}$ & $\begin{array}{r}106 \mathrm{~b} \\
\mathrm{~A} \\
135 \mathrm{~b}\end{array}$ & $\begin{array}{r}93 \mathrm{~b} \\
\mathrm{~A} \\
119 \mathrm{C}\end{array}$ & $\begin{array}{r}118 \mathrm{a} \\
\mathrm{A} \\
155 \mathrm{a}\end{array}$ & $\begin{array}{l}20,6 \\
25,9\end{array}$ \\
\hline 1985 & $\begin{array}{l}42 \\
53\end{array}$ & $\begin{array}{r}110 b \\
A \\
162 b\end{array}$ & $\begin{array}{r}105 \mathrm{C} \\
\mathrm{A} \\
154 \mathrm{C}\end{array}$ & $\begin{array}{r}97 \mathrm{~d} \\
\mathrm{~A} \\
135 \mathrm{~d}\end{array}$ & $\begin{array}{r}122 \mathrm{a} \\
\mathrm{A} \\
176 \mathrm{a}\end{array}$ & $\begin{array}{l}19,6 \\
22,4\end{array}$ \\
\hline
\end{tabular}

Les résultats de pesée d'œufs, obtenus sur quelques échantillons seulement, sont indiqués dans le tableau 7. Les poules M-88 pondent les œufs les plus gros (en moyenne 63,5 g) et les poules P-00 les œufs les plus petits (en moyenne $58,3 \mathrm{~g}$ ). On peut remarquer que la souche M-88 a un format plus réduit que celui des souches M-22 et $\mathrm{M}-33$, qu'elle possède le gène de nanisme $d w$, mais qu'elle donne des œufs plus gros. Cela résulte du pool génétique particulier de la population de départ (voir description de cette souche).

Les caractéristiques de sperme, mesurées de 1983 à 1985, sont détaillées dans le tableau 8. Le nombre de spermatozoides par éjaculat est plus élevé dans la souche $\mathrm{M}-33$. La souche $\mathrm{P}-00$ vient ensuite, puis les souches M-22 et M-88 qui sont équivalentes. Pour la note de motilité dont la plage de variation est grande (de 0 à 80 ), on n'observe pas de différence systématique entre les 4 souches. Ces caractéristiques présentent une

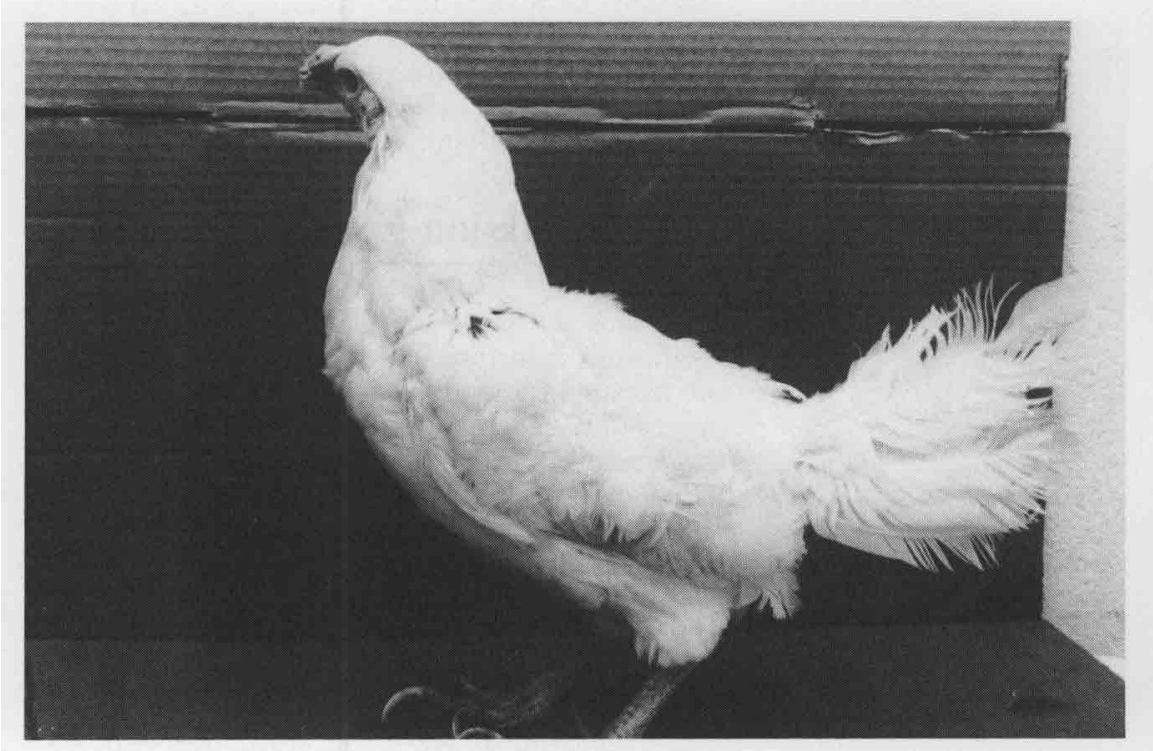

Tableau 6. Pourcentage d'œufs cassés (et anormaux) par rapport au nombre total d'cufs pondus depuis l'âge de 21 semaines pour l'ensemble des poules, par souche et par année d'éclosion.

\begin{tabular}{|c|c|c|c|c|l|}
\hline \multirow{2}{*}{$\begin{array}{c}\text { Année } \\
\text { d'éclosion }\end{array}$} & \multirow{2}{*}{$\begin{array}{c}\text { Age } \\
\text { (semaines) }\end{array}$} & \multicolumn{4}{|c|}{ Souche } \\
\cline { 3 - 6 } & & $\mathbf{M - 2 2}$ & $\mathbf{M}-\mathbf{3 3}$ & $\mathbf{M}-\mathbf{8 8}$ & \multicolumn{1}{|c|}{ P-00 } \\
\hline \multirow{2}{*}{1981} & 42 & $13,8 \mathrm{bD}(1)$ & $14,5 \mathrm{bE}$ & $16,3 \mathrm{CC}$ & $4,8 \mathrm{aA}$ \\
& 53 & $15,5 \mathrm{~b}$ & $15,2 \mathrm{~b}$ & $17,2 \mathrm{~b}$ & $6,0 \mathrm{a}$ \\
& 61 & $17,1 \mathrm{C}$ & $16,1 \mathrm{~b}$ & $18,0 \mathrm{~d}$ & $6,7 \mathrm{a}$ \\
1982 & 42 & $5,2 \mathrm{aB}$ & $8,6 \mathrm{bD}$ & $20,7 \mathrm{CD}$ & $4,8 \mathrm{aA}$ \\
1983 & 42 & $2,9 \mathrm{aA}$ & $5,2 \mathrm{bA}$ & $15,8 \mathrm{dC}$ & $6,3 \mathrm{cB}$ \\
& 53 & $2,9 \mathrm{a}$ & $5,4 \mathrm{~b}$ & $16,0 \mathrm{~d}$ & $7,0 \mathrm{C}$ \\
\multirow{2}{*}{1984} & 42 & $4,8 \mathrm{aB}$ & $6,4 \mathrm{bB}$ & $14,7 \mathrm{cB}$ & $4,8 \mathrm{aA}$ \\
& 50 & $5,4 \mathrm{~b}$ & $6,8 \mathrm{C}$ & $14,5 \mathrm{~d}$ & $4,8 \mathrm{a}$ \\
1985 & 42 & $7,5 \mathrm{bC}$ & $7,6 \mathrm{bC}$ & $9,8 \mathrm{cA}$ & $5,4 \mathrm{aA}$ \\
& 53 & $7,8 \mathrm{C}$ & $7,2 \mathrm{~b}$ & $9,4 \mathrm{~d}$ & $6,5 \mathrm{a}$ \\
\hline
\end{tabular}

(1) Le test $x^{2}$ a été utilisé pour comparer les souches ou les années. Pour une même ligne, deux valeurs suivies d'une même lettre minuscule ne sont pas sig nificativement différentes $(\mathrm{P}>0,05)$. L.es comparaisons entre années n'ont été faites qu'à l’âge de 42 semaines : pour une même colonne deux valeurs suivies de la même lettre majuscule ne sont pas significativement différentes $(\mathrm{P}>0,05)$. La lettre a (ou A) indique le résultat le plus favorable, c'est-à-dire le plus faible taux d'oufs cassés.

La proportion d'œufs cassés est très élevée pour la souche $M-88$. A partir de 1983, la solidité de la coquille a été améliorée en intégrant ce critère dans la sélection de cette souche. 
Tableau 7. Poids moyen de l'œuf (grammes) estimé à différents âges.

\begin{tabular}{|c|c|c|c|c|c|c|}
\hline \multirow{2}{*}{$\begin{array}{c}\text { (1) } \\
\text { Année } \\
\text { d'éclosion }\end{array}$} & \multirow{2}{*}{$\begin{array}{c}\text { Age } \\
\text { (semaines) }\end{array}$} & \multicolumn{4}{|c|}{ Moyennes } & \multirow{2}{*}{$\begin{array}{l}\text { Ecart-type } \\
\text { intra-souche }\end{array}$} \\
\hline & & M-22 & M- 33 & M- 88 & P-00 & \\
\hline 1981 & 53 & $68,3 \mathrm{a}$ & $64,8 \mathrm{~b}$ & 69,7 a & $62,1 \mathrm{C}$ & 4,61 \\
\hline 1983 & 35 & $57,1 \mathrm{C}$ & 59,4 a & 60,8 a & $57,9 \mathrm{~b}$ & 4,06 \\
\hline 1984 & 42 & $61,5 \mathrm{~b}$ & $61,4 \mathrm{~b}$ & 62,9 a & $57,0 \mathrm{C}$ & 4,00 \\
\hline 1985 & 42 & 59,7 & 61,1 & 60,4 & 56,2 & (2) \\
\hline
\end{tabular}

(1) Le poids de l'œuf n'a pas été mesuré pour les poules nées en 1982.

(2) Pesée globale par souche, l'écart-type des valeurs individuelles n'est donc pas disponible.

variabilité phénotypique élevée entre individus d'un même groupe élémentaire souche-année d'éclosion (coefficients de variation supérieurs à $50 \%$ ), ainsi que de grandes variations d'une année à l'autre (en particulier pour la note de motilité). On peut donc penser à une forte influence des conditions de la mesure, ce qui rend difficile l'interprétation des résultats. Pour les deux caractéristiques indiquées au tableau 8 , une analyse de variance à 2 facteurs, effectuée en prenant le même effectif unitaire $(n=30)$, a fait apparaître une interaction souche $\times$ année d'éclosion significative $(\mathrm{P}<0,05)$.

\section{3 / Discussion-Conclusion}

Les résultats que nous venons de détailler permettent d'avoir une idée de ce que peut donner un travail de conservation de souches de volailles mises dans des conditions proches de la pratique professionnelle. Nous discuterons d'une part les conséquences de la sélection opérée de 1981 à 1985 et d'autre part la valeur d'utilisation éventuelle de nos souches en les comparant aux croisements commerciaux.

\section{1 / Progrès réalisés de 1981 à 1985}

La pression de sélection appliquée peut être considérée comme "légère » puisque environ $50 \%$ des poules mises en contrôle de ponte étaient utilisées comme reproductrices. Une indication de la réponse directe à la sélection apparaît dans le tableau 1 qui donne les nombres d'œufs normaux contrôlés à l'âge de 42 semaines : les poules nées en 1985 ont pondu en moyenne 10 œufs de plus que celles nées en 1981 , soit un gain moyen de 2 cufs par an (environ $2 \%$ ). Cette amélioration n'est pas régulière selon les générations. On observe en effet une diminution du nombre d'œufs en 1982 pour la souche M-88 et en 1983 pour les 3 autres souches. Ces fluctuations peuvent résulter des aléas de l'environnement mais il n'est pas possible de préciser davantage puisque nous ne disposions pas de population témoin. La réponse directe à la sélection est également variable selon les souches: entre 1981 et 1985 le gain est approximativement de 13 œufs pour les souches M-22 et P-00, moins de 10 cufs pour la souche M-88 et seulement 7 œufs pour la souche M-33. Dans ce dernier cas, l'écart n'est pas statistiquement significatif.

Les autres caractères de ponte peuvent être considérés comme des réponses corrélées. Les évolutions entre 1981 et 1985 pour l'âge au premier œuf et la ponte à l'âge de 53 semaines sont visualisées dans la figure 3 . En moyenne, les poules pondent 9 jours plus tôt en 1985, soit un gain de $5 \%$, et le nombre total d'œufs a augmenté de 9 unités, soit un gain de $6 \%$. Quand on estime la production en intensité de ponte, le gain n'est que de $1 \%$ car les 9 ceufs supplémentaires ont été obtenus sur une période de temps plus longue. La comparaison des souches montre à nouveau que le progrès relatif de la ponte des poules M-33 a été faible : pour une précocité avancée de 10 jours on n'obtient que 4 oufs de plus à l'âge de 53 semaines, d'où une diminution de l'intensité de ponte. C'est dans la souche M-22 qu'on observe la plus forte augmentation du nombre d'œufs à 53 semaines d'âge (près de 16 entre 1981 et 1985), suivie par la souche P-00 (près de 13). Durant la même période, la fréquence des ceufs cassés a

Tableau 8.

Caractéristiques du sperme des coqs, mesurées sur 30 à 50 coqs par souche et par année.

\begin{tabular}{|c|c|c|c|c|c|}
\hline \multirow{2}{*}{ Année d'éclosion } & \multicolumn{4}{|c|}{ Moyennes par souche } & \multirow{2}{*}{$\begin{array}{c}\text { Ecart-type } \\
\text { intra-souche }\end{array}$} \\
\hline & $\mathrm{M}-22$ & M-33 & M-88 & P-00 & \\
\hline $\begin{array}{l}\text { 1) Nombre de spermatozoïdes } \\
\text { par éjaculat (en milliards) }\end{array}$ & & & & & \\
\hline 1983 & $1,78 b$ & $2,41 \mathrm{a}$ & $1,93 \mathrm{~b}$ & $1,59 \mathrm{~b}$ & 0,83 \\
\hline 1984 & $1,66 \mathrm{~b}$ & $2,14 \mathrm{a}$ & $1,51 \mathrm{~b}$ & $1,86 \mathrm{ab}$ & 0,79 \\
\hline 1985 & $1,35 \mathrm{~b}$ & $1,55 \mathrm{ab}$ & $1,25 \mathrm{~b}$ & $1,92 \mathrm{a}$ & 0,97 \\
\hline $\begin{array}{l}\text { 2) Note de motilité } \\
\text { (plage de variation : } 0-80 \text { ) }\end{array}$ & & & & & \\
\hline 1983 & $10 \mathrm{a}$ & $12 \mathrm{a}$ & 12 a & $9 \mathrm{a}$ & 5,4 \\
\hline 1984 & 30 a & 33 a & $37 \mathrm{a}$ & $32 \mathrm{a}$ & 16,5 \\
\hline 1985 & 24 a & $15 \mathrm{~b}$ & $16 \mathrm{~b}$ & $17 \mathrm{~b}$ & 11,8 \\
\hline
\end{tabular}


diminué en moyenne de plus de $40 \%$. Mais il faut souligner que cette fréquence était particulièrement élevée en 1981 pour les souches M-22, M-33 et M-88. Pour la souche P-00, la fréquence des œufs cassés a toujours été relativement faible et a peu évolué entre 1981 et 1985 . Cette faible fréquence peut être reliée à la présence du gène $O$ (œuf bleu) qu'on sait être associé à une coquille plus solide. Au contraire, les forts pourcentages d'œufs cassés observés dans la souche M-88 peuvent être associés au poids de l'œuf plus élevé dans cette souche, malg ré la présence du gène $d w$ qui par lui-même entraine un taux d'œuf cassés plus faible.

Pour les autres caractéristiques mesurées (poids vif, poids de l'œuf, caractéristiques du sperme), les données recueillies en 1981 el 1985 ne permeltent pas d'évaluer les progrès réalisés car elles sont soit incomplètes soit non comparables.

\section{2 / Comparaison avec les souches commerciales}

Cette comparaison a été faite en prenant comme référence les testages réalisés à la Station expérimentale d'aviculture de Ploufragan sur des animaux nés en 1983 : moyenne de toutes les souches (œufs colorés, œufs blancs et souches expérimentales) de la 24 "épreuve de ponte (L'Hospitalier et al 1984) et moyenne des souches "lourdes nanifiées" testées en cage de ponte de

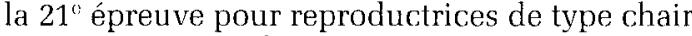
(Quemeneur et al 1984). Pour les souches du conservatoire, nous avons regroupé les moyennes des années 1983, 1984 et 1985, pour lesquelles nous avions le maximum de données comparables. Les valeurs utilisées pour les comparaisons sont indiquées dans le tableau 9 et ont été obtenues soit directement soit par intrapolation à partir des données des différentes périodes de ponte. La précocité de ponte a été estimée par l'âge à $50 \%$ de ponte. La production a été estimée à partir des caractéristiques au pic de ponte (âge des poules et intensité), de l'intensité de ponte aux âges de 30 et 50 semaines et du nombre d'œufs contrôlés à 1 an d'âge. Une estimation de la persistance est donnée par la diminution moyenne par semaine de l'intensité de ponte entre 30 et 50 semaines d'âge.

Les meilleures performances de ponte sont bien entendu observées sur les souches de pondeuses testées à Ploufragan. Cette supériorité s'explique par le fait que les souches du Magneraud sont des lignées pures dont le taux de consanguinité oscille entre 20 et $30 \%$, tandis que les souches testées à Ploufragan sont des croisements (donc consanguinité nulle) de lignées fortement sélectionnées sur les performances de ponte. Par ailleurs, les conditions d'élevage sont différentes, celles de Ploufragan étant mieux adaptées à une production d'œufs élevée. Les reproductrices chairs "nanifiées » testées à Ploufragan présentent une entrée en ponte tardive, comparée aux souches du Magneraud, mais une bonne intensité de ponte et un poids d'œuf élevé.

En comparaison avec les testages réalisés à Ploufragan, c'est la souche $\mathrm{P}-00$ qui se révèle être la meilleure pondeuse, avec une excellente per-
Figure 3. Evolution des performances par souche entre les cheptels éclos en 1981 et en 1985.

$$
\begin{array}{llll}
\text { M - 22 } & \text { M }-33 & \text { M }-88 & P-00
\end{array}
$$

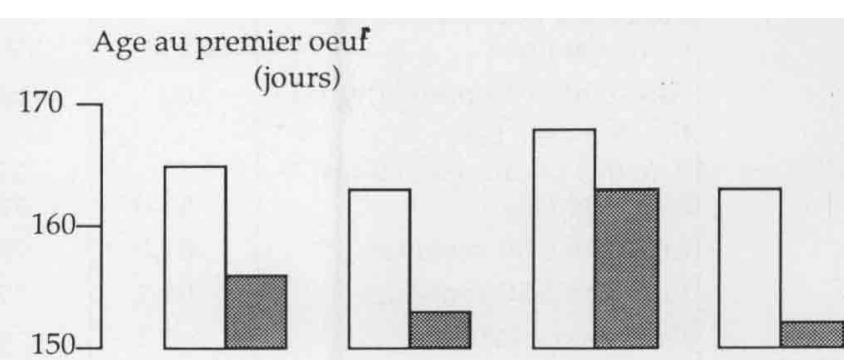

La souche P-00 présente les meilleures performances de ponte, mais avec un poids de l'auf plus faible que dans les autres souches.

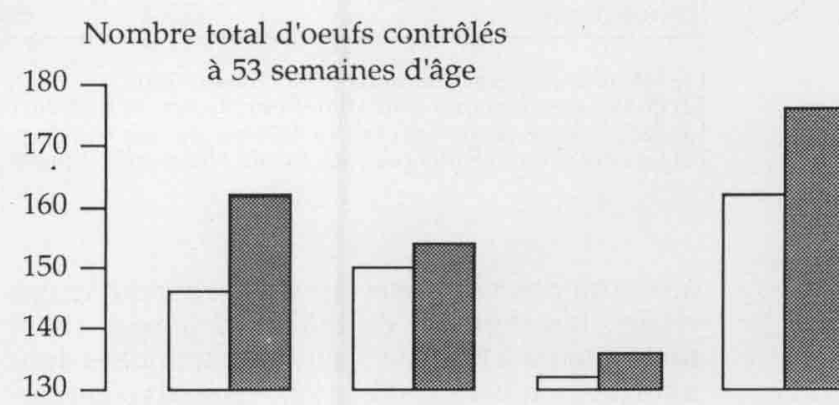

Intensité de ponte (\%)

à 53 semaines d'âge

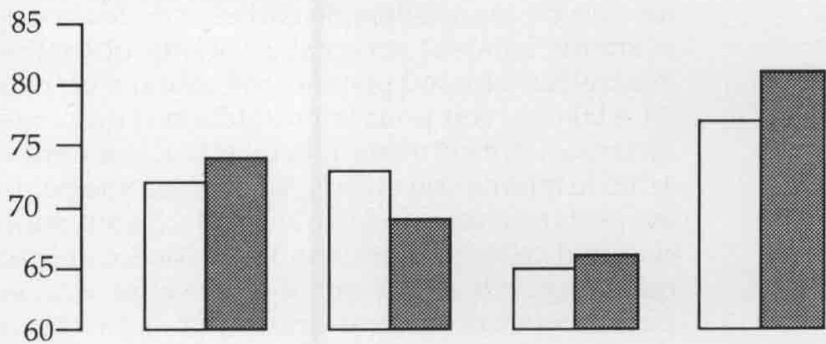

Pourcentage global d'oeufs cassés

à 53 semaines d'âge

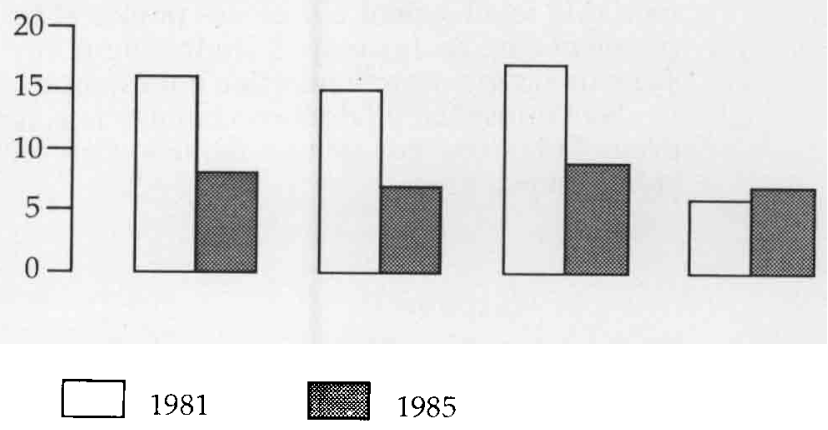

sistance mais aussi un poids d'œuf plus faible. Cette souche pourrait être intéressante pour des éleveurs voulant se démarquer des circuits commerciaux habituels grâce à la couleur bleue de l'œuf.

Les performances de ponte des souches M-22 et M-33 apparaissent compétitives comparées 
Tableau 9. Comparaison des souches du Magneraud avec les croisements testés à la Station de Ploufragan.

\begin{tabular}{|c|c|c|c|c|c|c|}
\hline \multirow[b]{2}{*}{ Caractéristiques } & \multicolumn{4}{|c|}{ Magneraud (1) } & \multicolumn{2}{|c|}{ Ploufragan } \\
\hline & M-22 & M-33 & M-88 & P-00 & $\begin{array}{c}24^{\mathrm{e}} \text { épreuve } \\
\text { ponte }\end{array}$ & $\begin{array}{l}21^{\mathrm{e}} \text { épreuve } \\
\text { chair (2) }\end{array}$ \\
\hline $\begin{array}{l}\text { Poids des poulettes à l'âge } \\
\text { de } 8 \text { semaines }\end{array}$ & 825 & 899 & 781 & 699 & 619 & 800 \\
\hline Age à $50 \%$ de ponte (jours) & 169 & 166 & 174 & 166 & 156 & 184 \\
\hline Pic de ponte (3) & & & & & & \\
\hline Age des poules (semaines) & 29 & 27 & 30 & 32 & 26 & 32 \\
\hline Intensité $(\%)$ & 83,0 & 75,6 & 72,5 & 83,1 & 92,6 & 80,5 \\
\hline Intensité à 30 semaines & 82,2 & 74,7 & 72,5 & 82,7 & 90,3 & 79,2 \\
\hline Intensité à 50 semaines & 61,1 & 57,4 & 49,9 & 70,9 & 78,0 & 57,6 \\
\hline Persistance $(4)$ & 1,1 & $\mathbf{0 , 9}$ & 1,1 & 0,6 & 0,6 & 1,1 \\
\hline Nombre d'œufs à 1 an & 149 & 142 & 132 & 166 & 179 & 135 \\
\hline $\begin{array}{l}\text { Poids ouf à } 40 \text { semaines } \\
\text { (grammes) }\end{array}$ & 59,4 & 60,6 & 61,4 & 57,0 & 60,1 & 62,8 \\
\hline
\end{tabular}

(1) Moyenne des 3 générations 1983, 1984 et 1985

(2) Poules de souches lourdes nanifiées placées en balterie de ponte.

(3) Estimation à partir des chiffres obtenus par période de ponte.

(4) Diminution moyenne par semaine de l'intensilé de ponte entre la $30^{\circ}$ et la $50^{\prime \prime}$ semaine.

aux souches commerciales de reproductrices chairs. Les données du tableau 2 (année 1982) montrent qu'à l'âge de 3 mois ces 2 souches donnent des poulets qui atteignent un poids commercial souvent recherché en France $(2 \mathrm{~kg}$ pour les coquelets et 1,5 kg pour les poulettes). Cet ensemble de résultats suggère la possibilité d'utiliser ces souches comme reproductrices chair, dans la mesure où les qualités de carcasse de leurs descendants seraient acceptables. Cette utilisation pourrait se faire soit pour la production d'un poulet « label», soit pour la production d'un poulet intermédiaire entre le « label » et le « standard », selon le type de coq utilisé. Au point de vue ponte, les performances de la souche M-22 sont supérieures à celles de la souche M-33. Mais cette dernière pourrait intéresser des éleveurs voulant commercialiser un œuf brun, c'est-à-dire beaucoup plus foncé que les œufs colorés des souches commerciales actuelles.

La souche M-88 présente a priori les caractéristiques les moins intéressantes : performances de ponte médiocres comparées aux souches commerciales et aux autres souches du Magneraud, mortalité relativement élevée des poules et fort pourcentage d'œufs cassés. Il faudrait donc envisager un programme de sélection important si on voulait l'utiliser en production commerciale, par exemple comme pondeuse "naine " d'un œuf suffisamment gros.

\section{Remerciements}

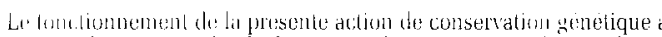
pu être réalisé grâce à l'aide financière du ministère de l'Agriculture Bureau des Productions liors-sol que nous remercions chaleureusement. De nombreuses personnes ont assumé la partie technique sans laquelle

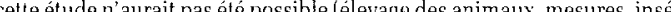

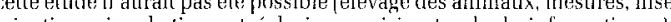
minaton, in Mesdames Y.Pern, I.K. Moeufet E. Veteat, Messienrs R, Barbin, P. Guillot, G. Malineau, M. Marchand, G. Marche. I'. Meteau, H. Plumet el J. Sarcy Nous tenons enfin à rendre un hommage particulier à feu le Dr L.P Cochez, ancien Dírecteur du Magneraud, qui a créé de nombreuses souches de poules dont les 4 maintenues en conservatoire. Son aide et ses conseils nous ont toujours étè précieux.

\section{Références bibliographiques}

ARNOLD I., de ROCI $\triangle$ MBEAU H., 1983. Inventaire des races menacées et des actions de conservation : les animaux de basse-cour. Ethnozootechnie, 33, 47-54.

BOYER J.P., 1965. De la création d'un conservatoire national de races françaises. C.R. 2' Conf. Avic. Furop., Bologne septembre 1964, Ed. Academia Nazionale di Agricoltura, Bologne, 399-408.

CAUDERON A., 1980. Sur la protection des ressources génétiques en relation avec leur surveillance, leur modelage et leur utilisation. C.R. Acad. Agric. Fr., 66 (12), 1051-1068.

COCHEZ L.P., 1951. Un facteur d'infertilité équilibré par la sélection chez la Wyandotte blanche. C.R. 9* Cong. Mondial Avic., Paris (Ed. Société Centrale Aviculture de France), Vol. 1, 122-128.

LHOSPITALIER R, QUFMENEUR P. LFMENECM, PROTAIS J., LAUNAY M., BOUGON M., 1984. Vingt-quatrième épreuve de ponte, 1983-1984. Bull. Inf. Stat. Exp. Avic. Ploufragan, 24, 112-150.

MERAT P., 1955. La race gatinaise : étude de sa variabilité génétique en vue d'obtenir des croisements homogènes: Ann. Zootechn., 4, 259-283.

MERAT P., 1962. Quelques relations entre caractères extérieurs à hérédité simple et productivité. Proc. 12th World's Poult. Cong., Sydney (Ed. Australian Branch W.P.S.A.), 7176.

MERAT P., 1972. Utilisation de facteurs mendéliens en élevage : l'exemple de la poule. Ann. Génét. Sél. anim., 4, 6777

PETITJEAN M.J., COCHEZ L.P., 1966. A propos de la subfertilité des coqs homozygotes pour le gène « $\mathrm{R}$ » (crête rosacée). C.R. 13" Cong. Mond. Avic., Kiev, (Ed. Branche Soviétique W.P.S.A.). 125-130.

QUEMENEUR P., L'HOSPITALIER R., LE MENEC M., DROUIN P., BOUGON M., 1984. Vingt-et-unième épreuve pour reproducteurs de type chair, 1983-1984. Bull. Inf. Stat. Exp. Avic. Ploufragan, 24, 163-201.

SILBERT J., MERAT P.., 1974. Etude génétique de la coloration bleue de la coquille des ceufs chez la poule : comparaison des performances des animaux Oo et oo. Ann. Génét. Sél. anim., 6, 405-414. 